\title{
Con: Can neuropathology really confirm the exact diagnosis?
}

Kurt A Jellinger*

\begin{abstract}
Clinical diagnostic accuracy using revised consensus criteria and newly developed biomarkers ranges from 65 to 96\% for Alzheimer's disease (AD), with a diagnostic specificity versus other dementias of 23 to $88 \%$. Neuropathological assessment using molecular biology and immunohistochemistry, homogeneous definitions, harmonized interlaboratory methods, and assessment standards can identify 54 to $97 \%$ of AD cases and can eliminate 62 to $100 \%$ of nondemented subjects, but only between 8 and $42 \%$ of non-AD dementias, without, however, being able to clarify the etiology of most of these disorders. The value and pitfalls of pathological diagnostic criteria are critically discussed.
\end{abstract}

\section{Introduction}

Dementia encompassing deteriorations in several cognitive domains can be caused by a large variety of disorders, disturbing brain functions due to loss of synapses and neurons. Consensus criteria for the clinical diagnosis of major dementing disorders exist and have recently been revised [1]. The combination of clinical data with biomarkers has improved the diagnostic accuracy of Alzheimer's disease (AD) from 65\% to between 92 and $96 \%$, while the sensitivity and specificity versus other dementias are much lower. Fusion of the best cerebrospinal fluid biomarkers and magnetic resonance imaging data will lead to a more precise diagnostic prediction [2].

Diagnostic guidelines for the neuropathological diagnosis of $\mathrm{AD}$ and other dementias rely on (semi)quantitative and topographic assessment of morphological and bio/histochemical signposts; in particular, specific protein inclusions in neurons and glia [3]. Diagnostic criteria for $\mathrm{AD}$ - in addition to cut-off values of senile plaques and tangles, their semiquantitative assessment

*Correspondence: kurt.jellinger@univie.ac.at

Institute of Clinical Neurobiology, 18 Kenyongasse, A-1070 Vienna, Austria and age adjustment in the Consortium to Establish a Registry for Alzheimer's Disease protocol - include the topographic staging of neuritic AD pathology, reevaluated recently [4].

The combination of the Consortium to Establish a Registry for Alzheimer's Disease and the Braak scores in the National Institute of Aging-Reagan Institute criteria relates dementia to AD typical lesions with high, intermediate and low likelihood [5]. Evaluation of the criteria showed their validity in $\mathrm{AD}$ - high lesion stages identifying 54 to $97 \%$ of $\mathrm{AD}$ cases and eliminating between 62 and $100 \%$ of nondemented subjects with low Braak stages, whereas only between 8 and $42 \%$ were identified among non-AD neurodegenerative dementias [1].

\section{Specific problems in the diagnosis of Alzheimer's disease}

The current algorithms for the neuropathological diagnosis of $\mathrm{AD}$, based on assessment of plaques and tangles, despite reasonable interrater agreement when using standardized criteria, only consider the classical plaque and tangle phenotype of AD but do not recognize other subtypes.

The plaque-predominant type with abundant amyloid plaques, with no or very little neuritic pathology restricted to the hippocampus and with abnormal phosphorylated tau in neocortical pyramidal cells, but lacking overt tangle formation, accounts for 3.5 to $8 \%$ of demented subjects over age 85 years [6]. Many of these cases are associated with cortical Lewy bodies, representing a specific type of dementia with Lewy bodies.

Tangle-predominant dementia occurring in the very old (age $80+$ years) and accounting for 5 to $7 \%$ of dementia cases shows tau pathology often restricted to the limbic system, an absence of neuritic plaques, and no or very little (diffuse) amyloid deposits. Since the tangles in this type react with three-repeat and four-repeat tau similar to those in classical AD, it could be considered a subtype of AD; tangle-predominant dementia, however, is clinically sometimes different and associated with different apolipoprotein E genotypes [7].

Standard metrics for plaques and tangles are usually semiquantitative; good agreement was reached only 
when the lesions were substantial in isocortical structures (Braak stage V to VI with absolute agreement 91\%), while for mild stages the agreement was poorer [8], limiting the ability to make accurate correlation of antemortem cognitive status and pathology. Although the sensitivity and specificity of the National Institute of Aging-Reagan Institute criteria are suggested to be $90 \%$, only 30 to $57 \%$ of the brains of patients with a clinical diagnosis of probable AD show pure AD pathology [1]. Their predictive value may thus be reduced to 38 to $44 \%$ [9]. In a retrospective clinicopathological study of 1,700 demented persons (66\% female; Mini-Mental State Examination score <20; mean age at death 84.3 to 6.0 years; $90 \%$ over age 70 years), AD-related lesions were present in $83.2 \%$, but pure $\mathrm{AD}$ without other pathologies was present in only $42.0 \%$, AD with other pathologies including mixed dementia in $41.2 \%$, vascular dementia in $12.8 \%$, other disorders in $4.1 \%$, and negative pathology in $0.9 \%$ [10].

Although cognitively unimpaired subjects may show variable neocortical AD pathology, and although good correlations between the severity and extension of tau pathology and/or of $\beta$-amyloid load have been found, at least in those without superimposed other brain diseases [11], the distinction between physiological and pathological aging (often but not consistently associated with cognitive decline) may be difficult. Specific problems arise from considerable differences between genetic/ familial AD and sporadic AD [12] and between oldest-old patients and younger patients, with considerable differences in both the intensity and distribution of $\mathrm{AD}$ pathology. Increased densities of neuritic plaques and tangles are absent in demented patients over age 90 years, with considerable overlap between demented and nondemented cases [13]. A high percentage of demented persons aged $80+$ years do not meet the pathological criteria of AD or were classified as dementia of unknown etiology [14]. In a prospective study of 180 demented patients (mean age $85 \pm 3.4$ years), autopsy showed AD in $48 \%$, AD with vascular pathology in $19 \%$, vascular dementia in $11 \%$, dementia with Lewy bodies in $9 \%$, and dementia of unknown etiology in 13\% (KA Jellinger, unpublished observations).

An important problem is the frequent presence of confounding processes in the aged brain that coexist with AD - such as cerebrovascular disease, Lewy body pathology, argyrophilic grain disease, hippocampal sclerosis, and so forth - with about two-thirds of cases showing mixed pathologies (see $[1,15]$ ), which have, however, frequently been missed clinically and could not be identified without neuropathological examination using modern biochemical and molecular-biological analyses $[3,16]$. Since 50 to $85 \%$ of the brains of oldest-old patients show cerebrovascular lesions, a specific problem is their impact in relation to AD pathology [15]. The burden of vascular and AD-type pathologies are considered independent of each other, and are consistent with an additive or synergistic effect of both types on cognitive impairment $[1,17]$. It should be borne in mind that all additional pathologies may interact, although their mutual impact often remains unclear.

There is increasing use of biochemical (and genetic) approaches for refinement of diagnosis and analysis of the relevant contribution of different disease processes to neurodegeneration of $\mathrm{AD}$ and other dementias $[1,3,16,18]$. Since the majority of degenerative dementing disorders are associated with intracellular and/or extracellular deposition of misfolded proteins (proteinopathies), most of them can be classified and diagnosed by morphological, immunohistochemical and/or molecular-biological (neurochemical) identification of these deposits representing characteristic markers and signposts of particular disorders. Algorithms for the molecular-pathological classification of sporadic (nongenetic/nonhereditary) forms of neurodegenerative dementias have been proposed recently $[3,16,18]$. Since there is considerable clinical and morphological overlap between many of these disorders, however, the reliability and clinical relevance of the current diagnostic criteria need better qualification and validation.

\section{Conclusion}

Although molecular genetics, biochemistry and animal models, at least in part reproducing the morphology of human $\mathrm{AD}$ and related disorders, have produced a large and convincing body of data on the pathogenesis and pathophysiology of the disease and have made an increasing contribution to postmortem studies of the cellular and molecular changes that underpin AD and other causes of dementia, the molecular backgrounds, the basic etiological factors, the pathogenic interrelationships of various concomitant pathologies, and the impact for an exact diagnosis of $\mathrm{AD}$ need further validation. Harmonized techniques are required to increase the accuracy and reproducibility of neuropathological diagnosis as a basis for further successful treatment and neuroprotection.

\footnotetext{
Abbreviations

$A D$, Alzheimer's disease.

Competing interests

The author declares that he has no competing interests.

Acknowledgements

The author thanks E Mitter-Ferstl, PhD, for secretarial assistance. The work was supported by the Society for the Support of Research in Experimental Neurology, Vienna, Austria.

Published: 7 May 2010

References

1. Jellinger KA: Criteria for the neuropathological diagnosis of dementing disorders: routes out of the swamp? Acta Neuropathol 2009, 117:101-110
} 
2. Jack CR, Jr, Knopman DS, Jagust WJ, Shaw LM, Aisen PS, Weiner MW, Petersen RC, Trojanowski JQ: Hypothetical model of dynamic biomarkers of the Alzheimer's pathological cascade. Lancet Neurol 2010, 9:119-128.

3. Kovacs GG, Botond G, Budka H: Protein coding of neurodegenerative dementias: the neuropathological basis of biomarker diagnostics. Acta Neuropathol 2010, 119:389-408.

4. Braak H, Alafuzoff I, Arzberger T, Kretzschmar H, Del Tredici K: Staging of Alzheimer disease-associated neurofibrillary pathology using paraffin sections and immunocytochemistry. Acta Neuropathol (Berl) 2006, 112:389-404.

5. Hyman BT, Trojanowski JQ: Consensus recommendations for the postmortem diagnosis of Alzheimer disease from the National Institute on Aging and the Reagan Institute Working Group on diagnostic criteria for the neuropathological assessment of Alzheimer disease. J Neuropathol Exp Neurol 1997, 56:1095-1097.

6. Tiraboschi P, Sabbagh MN, Hansen LA, Salmon DP, Merdes A, Gamst A, Masliah E, Alford M, Thal $\amalg$, Corey-Bloom J: Alzheimer disease without neocortical neurofibrillary tangles:'a second look'. Neurology 2004, 62:1141-1147.

7. Jellinger KA, Attems J: Neurofibrillary tangle-predominant dementia: comparison with classical Alzheimer disease. Acta Neuropathol 2007, 113:107-117.

8. Alafuzoff I, Arzberger T, Al-Sarraj S, Bodi I, Bogdanovic N, Braak H, Bugiani O, Del-Tredici K, Ferrer I, Gelpi E, Giaccone G, Graeber MB, Ince P, Kamphorst W, King A, Korkolopoulou P, Kovacs GG, Larionov S, Meyronet D, Monoranu C, Parchi P, Patsouris E, RoggendorfW, Seilhean D, Tagliavini F, Stadelmann C, Streichenberger N, Thal DR, Wharton SB, Kretzschmar H: Staging of neurofibrillary pathology in Alzheimer's disease: a study of the BrainNet Europe Consortium. Brain Pathol 2008, 18:484-496.

9. Bowler JV, Munoz DG, Merskey H, Hachinski V: Fallacies in the pathological confirmation of the diagnosis of Alzheimer's disease. J Neurol Neurosurg Psychiatry 1998, 64:18-24.
10. Jellinger KA, Attems J: Prevalence of dementia disorders in the oldest-old: an autopsy study. Acta Neuropathol 2010, 119:421-433.

11. Nelson PT, Abner EL, Schmitt FA, Kryscio RJ, Jicha GA, Smith CD, Davis DG, Poduska JW, Patel E, Mendiondo MS, Markesbery WR: Modeling the association between 43 different clinical and pathological variables and the severity of cognitive impairment in a large autopsy cohort of elderly persons. Brain Pathol 2010, 20:66-79.

12. Woodhouse A, Shepherd CE, Sokolova A, Carroll VL, King AE, Halliday GM, Dickson TC, Vickers JC: Cytoskeletal alterations differentiate presenilin-1 and sporadic Alzheimer's disease. Acta Neuropathol 2009, 117:19-29.

13. Haroutunian V, Schnaider-Beeri M, Schmeidler J, Wysocki M, Purohit DP, Perl DP, Libow LS, Lesser GT, Maroukian M, Grossman HT: Role of the neuropathology of Alzheimer disease in dementia in the oldest-old. Arch Neurol 2008, 65:1211-1217.

14. Crystal HA, Dickson D, Davies P, Masur D, Grober E, Lipton RB: The relative frequency of 'dementia of unknown etiology' increases with age and is nearly 50\% in nonagenarians. Arch Neurol 2000, 57:713-719.

15. Schneider JA, Arvanitakis Z, Bang W, Bennett DA: Mixed brain pathologies account for most dementia cases in community-dwelling older persons. Neurology 2007, 69:2197-2204

16. Dickson DW: Neuropathology of non-Alzheimer degenerative disorders. Int J Clin Exp Pathol 2010, 3:1-22. [www.ijcep.com/IJCEP908006]

17. Duyckaerts C, Delatour B, Potier MC: Classification and basic pathology of Alzheimer disease. Acta Neuropathol 2009, 118:5-36.

18. Jellinger KA: Basic mechanisms of neurodegeneration: a critical update. J Cell Mol Med 2010, 14:457-487.

doi:10.1186/alzrt34

Cite this article as: Jellinger KA: Con: Can neuropathology really confirm the exact diagnosis? Alzheimer's Research \& Therapy 2010, 2:11. 